Published in final edited form as:

J Phys Chem B. 2016 July 7; 120(26): 6102-6109. doi:10.1021/acs.jpcb.6b02079.

\title{
Translocation of an Incompressible Vesicle through a Pore
}

\author{
Hamid R. Shojaei ${ }^{\dagger}$ and Murugappan Muthukumar ${ }^{*}, \ddagger$ \\ tDepartment of Physics, University of Massachusetts, Amherst, Massachusetts 01003, United \\ States \\ FDepartment of Polymer Science and Engineering, University of Massachusetts, Amherst, \\ Massachusetts 01003, United States
}

\begin{abstract}
We have derived the free energy landscape for the translocation of a single vesicle through a narrow pore by accounting for bending and stretching of the vesicle, and the deformation of the vesicle by the pore. Emergence of a free energy barrier for translocation is a general result, and the magnitude of the barrier is calculated in terms of the various material parameters. The extent of the reduction in the barrier by the presence of an external constant force is calculated. Using the Fokker-Planck formalism, we have calculated the average translocation time corresponding to the various free energy landscapes representing different parameter sets. The dependencies of the average translocation time on the strength of the external force, vesicle size, bending and stretching moduli of the vesicle, and radius and length of the pore are derived, and the computed results are discussed.
\end{abstract}

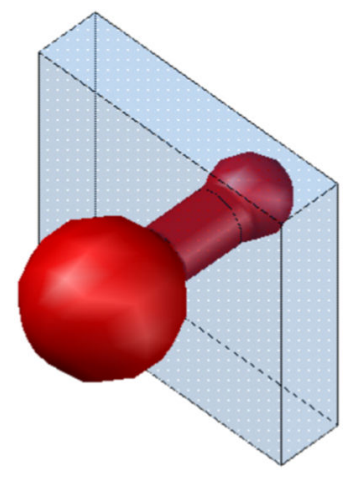

\section{INTRODUCTION}

The passage of vesicles through a small pore is pertinent to many transdermal applications ${ }^{1,2}$ and drug delivery. ${ }^{2,3}$ While the specifics of the process are quite diverse in terms of chemical

\footnotetext{
*Corresponding Author muthu@polysci.umass.edu. ASSOCIATED CONTENT

Supporting Information

The Supporting Information is available free of charge on the ACS Publications website at DOI: 10.1021/acs.jpcb.6b02079.

Geometrical calculations for partial sphere, cone, cylinder, and partial torus (PDF)

The authors declare no competing financial interest.
} 
details of the various particular systems and the driving forces, the general features have recently been addressed by using statistical mechanical description. ${ }^{4-9}$ It is now generally recognized that the fluctuations of the shape of vesicles are controlled by membrane curvature, tension, and interactions with the environment such as colloidal beads, ${ }^{9-19}$ macromolecules, ${ }^{20}$ and pores. ${ }^{8,9,21}$ Coarse-grained parametrization of specific details regarding chemical compositions of the vesicles into universal mechanical features such as bending and stretching has generated considerable insight into the behavior of such intrinsically complex systems. One of the pioneering works using this approach is by Deserno and Gelbart, ${ }^{10}$ who treated the phenomenon of endocytosis by considering the wrapping of a spherical particle by a vesicle. In this paper, we address the passage of a vesicle through a narrow pore by adopting the same model as that of Deserno and Gelbart. ${ }^{10}$

Basically, the shape fluctuations of a free vesicle are described by a bending modulus $\boldsymbol{x}_{\mathrm{c}}$ and a stretching modulus $\lambda$, with the free energy $F_{\mathrm{H}}$ of the vesicle given by

$$
F_{\mathrm{H}}=\frac{1}{2} \kappa_{\mathrm{c}} \oint d A\left(2 H-c_{0}\right)^{2}+\frac{1}{2 A_{0}} \lambda\left(A-A_{0}\right)^{2}
$$

The first term on the right-hand side of eq 1 gives the bending energy in the form of the Helfrich free energy ${ }^{22}$ as harmonic in $H$, which is the mean curvature of the membrane for a given vesicle geometry. This is given by $H=\frac{1}{2}\left(\frac{1}{r_{1}}+\frac{1}{r_{2}}\right)$, where $r_{1}$ and $r_{2}$ are the principal radii at a point on the surface. The spontaneous curvature $c_{0}$ is a constant at all points on the vesicle and arises from asymmetry in the areas of the inner and outer surfaces of the lipid bilayer constituting the membrane of the vesicle. ${ }^{22-26}$ The integral over the surface area of $\left(2 H-c_{0}\right)^{2}$ gives the energy penalty associated with creating different curvature from the spontaneous curvature. ${ }^{4}$ This asymmetry then results in a curvature in the membrane even in the absence of external forces. The energy penalty to bend a vesicle is then with reference to this spontaneous curvature. The integral extends over the whole vesicle surface. The second term on the right-hand side of eq 1 is the stretching free energy penalty. This is usually taken to be quadratic in the change of the vesicle area $\Delta A=A-A_{0}$, where $A_{0}$ is the initial area of the vesicle and $A$ is that of the perturbed vesicle.

The trajectory of a successful translocation of a vesicle from a donor compartment into a receiver compartment through a narrow pore is sketched schematically in Figure 1. The free energy penalty associated with bending and stretching of the vesicle in going from state a to state e in Figure 1 is expected to result in a free energy barrier for the translocation process. ${ }^{8}$ The emergence of free energy barriers for translocation processes is a ubiquitous phenomenon, as seen even for one-dimensional topological structures such as linear polymers. ${ }^{27}$ If an external force sufficiently strong to enable the vesicle to cross the barrier is applied to the vesicle, successful translocation events will occur. The speed of translocation depends on the strength of the applied external force.

The primary goal of the present paper is to assess the dependence of the free energy barrier and the translocation time as functions of the material parameters $\kappa_{\mathrm{c}}$ and $\lambda$, the strength of the external force which is taken to arise from a pressure gradient, and the feature sizes of 
the vesicle and pore. The particular details of specific vesicles in terms of their chemical composition and biological contexts are treated only through the material parameters used in the present coarse-grained model. Here we restrict our calculations to incompressible spherical vesicles of radius $r_{0}$ (in states a and e of Figure 1) and cylindrical pores of radius $d$ and length $L$. Following the work of Deserno and Gelbart ${ }^{10}$ and our earlier work, ${ }^{20}$ we use a simple geometrical ansatz for the vesicle shape and calculate the free energy landscape for vesicle translocation. On the basis of the thus derived free energy profile, we use the Fokker-Planck formalism ${ }^{20,28}$ to compute the translocation rates in terms of the various parameters of the model.

The rest of the paper is organized as follows. In section II, we introduce the model and the procedures to calculate the free energy landscape and the translocation time. The main results are discussed in section III, followed by main conclusions in section IV.

\section{MODEL AND CALCULATIONS}

We assume that the vesicle is incompressible with volume $V_{0}=4 \pi r_{0}{ }^{3} / 3$ and that only the area of the vesicle changes during the translocation process through a cylindrical pore of radius $d$ and length $L$. We define the parameter $\beta$ as the ratio of the volume of the pore to that of the vesicle, $\beta=V_{\mathrm{p}} / V_{0}$, where $V_{\mathrm{p}}=\pi d^{2} L$. As sketched in Figure 1, there are three stages of translocation between the initial state (Figure 1a) and the final state (Figure 1e): (i) pore-filling stage, (ii) crossing stage, and (iii) pore-depletion stage. In order to describe the whole passage of the vesicle from the donor compartment to the receiver compartment, we define the translocation coordinate as $a=V_{\mathrm{t}} / V_{0}$, where $V_{\mathrm{t}}$ is the volume of the vesicle that is either inside the pore or that has passed through the pore. During the pore-filling stage, $V_{\mathrm{t}}$ is the same as the volume of the vesicle inside the pore $(0<a<\beta)$. During the crossing stage, the translocation coordinate represents the volume of the vesicle that is depleted from the donor compartment. When the vesicle is completely deleted from the donor compartment, $a=1$. From then onward, the depletion of the vesicle from the pore begins. For complete translocation, the vesicle must be fully transferred out of the pore. During the pore-depletion stage, $V_{\mathrm{t}}$ is the sum of the volume of the vesicle in the receiver compartment and the total volume of the pore. Therefore, we extend the domain of the translocation coordinate $a$ (defined above as the fraction of the volume of the vesicle that is removed from the donor compartment) beyond 1 to $1+\beta$ for the full translocation event. If the length of the pore is zero, this extended domain for the translocation coordinate is unnecessary.

We follow the procedure of Deserno and Gelbart ${ }^{10}$ and Mirigian and Muthukumar ${ }^{20}$ to calculate the free energy penalty associated with bending and stretching of the vesicle that accompany the translocation coordinate. When an additional curvature is demanded on the vesicle at the pore entrance (and at the pore exit), we introduce a torus at the contact point between the vesicle and pore mouth, as illustrated in Figure 2. To begin with, the free energy of the isolated vesicle in the initial state is given by eq 1 as

$$
\begin{aligned}
F_{0 \mathrm{~b}} & \left.\left.=\frac{\kappa_{\mathrm{c}}}{2}\left[4 \pi r_{0}{ }^{2}\left(\frac{2}{r_{0}}-c_{0}\right)^{2}\right)\right]=\frac{\kappa_{\mathrm{c}}}{2}\left[4 \pi\left(2-r_{0} c_{0}\right)^{2}\right)\right] \\
F_{0 \mathrm{~s}} & =0
\end{aligned}
$$


where $F_{0 \mathrm{~b}}$ and $F_{0 \mathrm{~s}}$ denote the bending and stretching contributions, respectively. This is also the result in the final state of translocation (Figure 1e). The free energy landscape for the three stages of translocation is derived next.

\section{Filling Stage}

As shown in Figure 2a, the additional curvature of the vesicle at the pore entrance or exit is obtained by introducing a torus at the contact point between the vesicle and the pore boundaries. This geometrical construction is made to generate smooth curvature variation along the translocation process. There are four components joined together in this state. First, we have a partial spherical shape, that is like a full sphere with a missing angle $\theta$. Next, there is a toroidal part that connects the sphere part to the pore. The third component is a cylinder with radius $d$ and length $I<L$. Finally, we have a hemisphere with radius $d$.

We define three parameters on the sphere-toroid part and $l$ as the length of the pore that is filled by vesicle. The radius of the partial sphere is $r$ which is smaller than $r_{0}$. The inner and outer radii of the torus are respectively $d$ and $d+2 b$ with the angle between the line connecting the center of the torus (pore) to the center of the sphere and the one from the center of the sphere and the circular part of toroid is $\theta$ (see Figure 2a). The change of the curvature should be continuous through the connecting point of the toroid and sphere. This gives us a relation between $r, \theta$, and $b$

$$
\begin{aligned}
\sin \theta & =\frac{b+d}{r+b} \\
b & =\frac{r}{1-\sin \theta}
\end{aligned}
$$

Since the vesicle is incompressible, the volume of the vesicle inside the pore is $a V_{0}$ and the volume of the vesicle in the donor compartment is $V_{\text {donor }}=(1-a) V_{0}$. The first definition gives us a relation for 1

$$
\begin{aligned}
\pi d^{2} l+\frac{2}{3} \pi d^{3} & =\alpha V_{0}=\frac{4}{3} \pi \alpha r_{0}{ }^{3} \\
l & =\frac{2}{3} d\left(2 \alpha \frac{r_{0} 3}{d^{3}}-1\right)
\end{aligned}
$$

The second condition gives us a constraint. We can find the total volume as a function of $r$, and $\theta$, for any value of $a$ by using eq 3 . Geometrical consideration gives the volume to be equal to the volume of a sphere with a missing angle $\theta$ plus a conical shape with radius at the bottom of the cone as $r+b$, and angle $\theta$, and subtracting the volume of partial toroid with inner radius $b$, and outer radius $d+2 b$, between angles $\frac{1}{2} \pi+\theta$ and $\pi$. Each part is independently calculated in the Supporting Information. The expression for the volume of the vesicle in the donor compartment is

$$
\begin{aligned}
V_{\text {donor }} & =(1-\alpha) V_{0}=V_{\mathrm{I}, \text { sph }}+V_{\mathrm{I}, \text { cone }}-V_{\mathrm{I}, \text { tor }} \\
& =\left[\frac{2}{3} \pi r^{3}(1+\cos \theta)\right]+\left[\frac{1}{3} \pi(b+d)^{3} \cot \theta\right]-\left[\pi\left[(b+d) b^{2}\left(\frac{\pi}{2}-\theta\right)-\frac{2}{3} b^{3} \cos \theta\right]\right]
\end{aligned}
$$


with $b$ defined in eq 3 . The solution to this equation gives $r(\theta)$, and we use the result in writing the free energy for each given $a$. After that, we minimize the free energy with respect to $\theta$.

Next we calculate the bending free energy term in the Helfrich free energy (eq 1)

$$
F_{\mathrm{I}, \text { bending }}=F_{\mathrm{I}, \mathrm{b}, s p h}+F_{\mathrm{I}, \mathrm{b}, \text { tor }}+F_{\mathrm{I}, \mathrm{b}, c y l}+F_{\mathrm{I}, \mathrm{b}, \text { hem }}
$$

Using the Supporting Information, the first term is

$$
\begin{aligned}
F_{1, \mathrm{~b}, s p h} & =\frac{1}{2} \kappa_{\mathrm{c}} \int d A\left(2 \frac{1}{r}-c_{0}\right)^{2} \\
& =\frac{1}{2} \kappa_{\mathrm{c}} 2 \pi r^{2}\left(2 \frac{1}{r}-c_{0}\right)^{2} \\
& =\frac{1}{2} \kappa_{\mathrm{c}} 2 \pi\left(2-c_{0} r\right)^{2}(1+\cos \theta)
\end{aligned}
$$

The second term is

$$
F_{\mathrm{I}, \mathrm{b}, \text { tor }}=\frac{1}{2} \kappa_{\mathrm{c}}\left[-2 \pi\left(c_{0} b+2\right)^{2} \cos (\theta)+2 \pi c_{0}\left(c_{0} b+2\right)\left(\frac{\pi}{2}-\theta\right)(b+d)+\frac{2 \pi(b+d)^{2}}{b \sqrt{d} \sqrt{2 b+d}}\left\{\pi-2 \tan ^{-1}\left(\frac{\sqrt{d}}{\sqrt{2 b+d}} \tan \left[\frac{1}{2}\left(\theta+\frac{\pi}{2}\right)\right]\right)\right\}\right]
$$

The next two terms in eq 6 are easy to find as

$$
\begin{array}{r}
F_{\mathrm{I}, \mathrm{b}, \mathrm{cyl}}=\frac{1}{2} \kappa_{\mathrm{c}} \pi d^{2} l\left(\frac{1}{d}-c_{0}\right)^{2} \\
=\frac{1}{2} \kappa_{\mathrm{c}} \frac{2}{3} \pi\left(2 \alpha \frac{r_{0}{ }^{3}}{d^{3}}-1\right)\left(1-c_{0} d\right)^{2} \\
F_{\mathrm{I}, \mathrm{b}, \text { hem }} \\
=\frac{1}{2} \kappa_{\mathrm{c}} 2 \pi d^{2}\left(2 \frac{1}{d}-c_{0}\right)^{2} \\
=\frac{1}{2} \kappa_{c} 2 \pi\left(2-c_{0} d\right)^{2}
\end{array}
$$

To calculate the stretching free energy term in the Helfrich free energy, eq 1, we need to find the change in the total area 


$$
\begin{aligned}
\Delta A_{\mathrm{I}} & =A_{\mathrm{I}}-A_{0} \\
& =A_{\mathrm{I}, s p h}+A_{\mathrm{I}, t o r}+A_{\mathrm{I}, c y l}+A_{\mathrm{I}, h e m}-A_{0} \\
& =\left[2 \pi r^{2}(1+\cos \theta)\right]+\left[2 \pi b\left[(b+d)\left(\frac{\pi}{2}-\theta\right)-b \quad \cos \theta\right]\right]+\left[2 \pi d^{2}\left(\frac{4}{3} \alpha \frac{r_{0}{ }^{3}}{d^{3}}-\frac{2}{3}\right)\right]+\left[2 \pi d^{2}\right]-\left[4 \pi r_{0}{ }^{2}\right]
\end{aligned}
$$

Therefore, the second term in eq 1 is

$$
F_{\mathrm{I}, \text { stretching }}=\frac{1}{2 A_{0}} \lambda\left(\Delta A_{\mathrm{I}}\right)^{2}
$$

where $A_{0}=4 \pi r_{0}^{2}$ and $\Delta A_{\mathrm{I}}$ is defined in eq 11 .

\section{Crossing Stage}

This stage corresponds to the state where the vesicle is on both sides of the pore (see Figure 1b) with $\beta<a<1\left(\beta=V_{\mathrm{p}} / V_{0}\right)$. The calculation is similar to the filling stage, but we have similar conditions for the receiver compartment side as well. We define new primed parameters which refer to the receiver side $\left(r^{\prime}, b^{\prime}\right.$, and $\left.\theta^{\prime}\right)$. While $b$ is defined in eq $3, b^{\prime}$ is

$$
\begin{aligned}
\sin \theta^{\prime} & =\frac{b^{\prime}+d}{r^{\prime}+b^{\prime}} \\
b^{\prime} & =\frac{r^{\prime} \sin \theta^{\prime}-d}{1-\sin \theta^{\prime}}
\end{aligned}
$$

Also, we have two separate conditions corresponding to the donor and receiver sides

$$
\begin{aligned}
V_{\text {donor }} & =(1-\alpha) V_{0}=V_{\text {sph }}+V_{\text {cone }}-V_{\text {tor }} \\
V_{\text {receiver }} & =\left(1-\alpha^{\prime}\right) V_{0}=V_{\text {sph }}^{\prime}+V_{\text {cone }}^{\prime}-V_{\text {tor }}^{\prime}
\end{aligned}
$$

with $(1-a)+\left(1-a^{\prime}\right)+\beta=1$, or $a^{\prime}=1+\beta-a$. Similar to the filling stage, these give us $r$ and $r^{\prime}$ as functions of $\theta$ and $\theta^{\prime}$, respectively.

The bending energy in this stage is in the form

$$
F_{I I, \text { bending }}=F_{I I, \mathrm{~b}, \text { sph }}+F_{I I, \mathrm{~b}, \text { tor }}+F_{I I, \mathrm{~b}, \text { pore }}+F_{I I, \mathrm{~b}, \text { sph }}^{\prime}+F_{I I, \mathrm{~b}, \text { tor }}^{\prime}
$$

$F_{\mathrm{II}, \mathrm{b}, \mathrm{sph}}$ and $F_{\mathrm{II}, \mathrm{b}, \mathrm{tor}}$ are defined in eqs 7 and 8. Also, $F_{I I, \mathrm{~b}, s p h}^{\prime}$ and $F_{I I, \mathrm{~b}, \text { tor }}^{\prime}$ are similar to substitutions $x \rightarrow x^{\prime}(x \equiv r, b, \theta)$. FII,b,pore is 


$$
\begin{aligned}
F_{I I, \mathrm{~b}, \text { pore }} & =\frac{1}{2} \kappa_{\mathrm{c}} 2 \pi L d\left(\frac{1}{d}-c_{0}\right)^{2} \\
& =\frac{1}{2} \kappa_{\mathrm{c}} 2 \pi \beta \frac{4}{3} \frac{r_{0}{ }^{3}}{d^{3}}\left(1-c_{0} d\right)^{2}
\end{aligned}
$$

with ${ }_{\mathrm{L}}$ being the total pore length and $\beta$ being the volume of the pore divided by the volume of the vesicle.

Calculation of area is also similar to that for the filling stage with the addition of terms from the receiver side

$$
\begin{aligned}
& \Delta A_{I I}=A_{I I}-A_{0} \\
& =A_{I I, s p h}+A_{I I, t o r}+A_{I I, p o r e}+A_{I I, s p h}^{\prime}+A_{I I, t o r}^{\prime}-A_{0} \\
& =\left[2 \pi r^{2}(1+\cos \theta)\right]+\left[2 \pi b\left[(b+d)\left(\frac{\pi}{2}-\theta\right)-b \quad \cos \theta\right]\right]+\left[2 \pi r^{\prime 2}\left(1+\cos \theta^{\prime}\right)\right]+\left[2 \pi b^{\prime}\left[\left(b^{\prime}+d\right)\left(\frac{\pi}{2}-\theta^{\prime}\right)-b^{\prime} \quad \cos \theta^{\prime}\right]\right]+
\end{aligned}
$$

and

$$
F_{I I, \text { stretching }}=\frac{1}{2 A_{0}} \lambda\left(\Delta A_{I I}\right)^{2}
$$

\section{Depletion Stage}

This is exactly analogous to the filling stage with the substitution of $x$ by $x^{\prime}(x \equiv r, b, \theta)$. Therefore, the result for this stage is given by eqs $6-12$ by replacing $r, b$, and $\theta$ by $r^{\prime}, b^{\prime}$, and $\theta^{\prime}$, respectively.

\section{External Driving Force}

In the absence of any external driving force, the free energy landscape is symmetric between the donor and receiver compartments, separated by a barrier as shown in section III (Figure 3). In order for the vesicle to undergo a net successful translocation from the donor compartment to the receiver compartment, the free energy of the system after translocation must be lower than that before translocation. This downhill free energy landscape is provided by an externally imposed driving force. The nature of the driving force depends on the specifics of the system. One example is the use of an externally imposed electric field to push charged vesicles or charged microgels through pores. An analogous situation arises for translocation of charged polymers such as DNA and proteins through nanopores. ${ }^{27} \mathrm{By}$ repeating the procedures outlined in ref 27 , it is in principle possible to derive the free energy contribution from such an externally imposed electric field on a vesicle bearing surface charges. The electrostatics of curved vesicle-fluid interfaces inside the pore is also quite complicated. We do not address the translocation of charged vesicles in this paper. Another example of the external force to drive the vesicle from the donor compartment to 
the receiver compartment is using a pressure gradient, as considered in an analogous problem by Linke et al. ${ }^{9}$ By maintaining a higher pressure in the donor compartment relative to the receiver compartment, the vesicle is pushed into the receiver compartment. The mechanics of elastic surfaces is very complex and in particular at the vesicle-fluid interface when a vesicle is partially filled inside a pore across a pressure gradient. Nevertheless, the essential physics is that when an incompressible vesicle of volume $V_{0}$ is pressed by an external pressure $P_{1}$ in the donor compartment into the receiver compartment with pressure $P_{2}$ through an orifice, the free energy changes from $P_{1} V_{0}$ to $P_{2} V_{0}$. Since $P_{1}>P_{2}$, the free energy landscape is downhill. The elastic distortion associated with the intermediate states of translocation, resulting in the barrier between the initial and final states, is treated as above. For pores of finite length, there is an additional friction between the vesicle and the pore wall, which is ignored. Therefore, the zeroth-order model of pressure-driven external contribution to free energy is

$$
F_{\text {ext }}=P\left(V_{0}-V_{\mathrm{t}}\right)
$$

where $V_{\mathrm{t}}$ is the volume of the incompressible vesicle that is depleted from the donor compartment and $P_{1}=P$ with $P_{2}=0$. An equivalent form for the external free energy has previously been used by Linke et al., ${ }^{9}$ where $P$ is taken as the osmotic energy difference between the donor and receiver compartments. Since the starting free energy value in constructing the free energy landscape is taken as zero, the constant $P V_{0}$ is absorbed in defining the reference state so that

$$
\Delta F_{\text {ext }}=-P V_{\mathrm{t}}
$$

Using the definition of $a=V_{\mathrm{t}} / V_{0}$ and multiplying and dividing by the constant $1+\beta$, where $\beta=V_{\mathrm{p}} / V_{0}$, the above equation is rewritten as

$$
\Delta F_{e x t}=-f_{0} \frac{\alpha}{1+\beta} \quad 0<\alpha<1
$$

where $f_{0}$ is the energy term $P V_{0}(1+\beta)$. We take the above equation for $0<a<1$. At $a=1$, the vesicle is completely depleted from the donor compartment. From now on, for the rest of the translocation process, we take $\Delta F_{\mathrm{ext}}=-f_{0} /(1+\beta)$.

Adding this term to the Helfrich free energy, we get the total free energy of the system as

$$
F=F_{\mathrm{H}}+F_{\text {ext }}=F_{\text {bending }}+F_{\text {stretching }}+\Delta F_{\text {ext }}
$$

for each of the three stages described above. 


\section{Kinetics of Translocation}

Now that we have the free energy landscape as a function of the translocation coordinate $a$, we follow the procedure of the Fokker-Planck formalism given in ref 20 and obtain the mean translocation time as the mean first passage time given by

$$
\tau=\frac{1}{k_{0}} \int_{0}^{1+\beta} \mathrm{d} \alpha_{1} \int_{0}^{\alpha_{1}} \mathrm{~d} \alpha_{2} \mathrm{e}^{\left[F\left(\alpha_{1}\right)-F\left(\alpha_{2}\right)\right] / k_{\mathrm{B}} T}
$$

In using this equation, we have assumed that the translocation time is longer than the relaxation time for the vesicle to relax from any distortion from its equilibrium state, by invoking the standard detailed balance arguments behind the Fokker-Planck formalism. Furthermore, we have solved the corresponding equation for the time-dependent probability distribution with the absorption boundary condition at the pore exit facing the receiver compartment and the reflecting boundary condition at the pore entrance facing the donor compartment. This set of boundary conditions is chosen for the present problem where there is a pressure driving the vesicle from the donor compartment to the receiver compartment. In the above equation, $k_{0}$ is a parameter representing local friction of a surface element of the vesicle as it goes through the pore and this is taken to set the unit of time for the problem. $k_{0}$ is a microscopic and nonuniversal parameter. The other parameters in the calculation are $\frac{r_{0}}{d}$, $\lambda, \kappa_{\mathrm{c}}, c_{0}, f_{0}$, and $\beta$. In the present paper, we report the results mainly for $c_{0}=0$ and $\beta=0.3$ as illustrative examples. We define the unit of energy as $k_{\mathrm{B}} T$, and $d$ is the unit of length. We use values for the rest of the parameters from different experiments. ${ }^{29-31}$ We have taken $\kappa_{\mathrm{c}}$ $\approx 2-5, \lambda \approx 1-10, r_{0} / d \approx 1.5-3$, and $d=1$, and $f 0$ is defined in such a way that the total translocation time will be between 10 and $10^{5}$ in units of $k_{0}{ }^{-1}$. The results given in the following section for these values of the parameters can readily be extended to other values of the parameters appearing in the free energy expressions.

\section{RESULTS AND DISCUSSION}

\section{Translocation Barrier}

Representative results for the free energy profile as a function of the translocation coordinate $a$ in the absence of any external driving potential difference are given in Figure 3. To calculate the free energy at any given $a$, we first find $r$ as a function of $\theta$ from the cubic root of the volume constraint, eq 5. Only one of the roots satisfies the conditions $0<\theta<\pi / 2$ and $d<r<r_{0}$. Substituting $I(\theta)$ in the Helfrich free energy, eq 1, we obtain the free energy as a function of $\theta$. Next, we minimize this free energy with respect to $\theta$ for the values of $\sin ^{-1}\left(d / r_{0}\right)<\theta<\pi / 2(\sin \theta$ cannot be smaller than the pore radius divided by the initial radius of the sphere). We consider values of $a$ from 0 to $1+\beta$ at increments of $\delta=0.01$. Finally, we get the free energy landscape of the system by shifting the free energy by a constant so that the initial free energy is 0 . Here the radius of the initial vesicle is 2.5 times the pore radius, the bending modulus is $2 k_{\mathrm{B}} T$, and the stretching modulus is $5 k_{\mathrm{B}} T$ per square of the pore radius. We have presented the results in Figure 3 with and without spontaneous curvature. The three stages of translocation are also marked on the figure. In general, there is a free energy barrier for the translocation process. The presence of spontaneous curvature 
reduces the barrier, the effect being larger in the crossing stage. Naturally, the free energy landscape is symmetric in $a$, as there is no driving potential difference between the initial and final states for Figure 3.

The role of the externally imposed potential difference is illustrated in Figure 4 for the same parameters as in Figure 3, when there is no spontaneous curvature. We follow the same method to numerically calculate the free energy as described above for $f_{0}=0$. For the driving potential difference $f_{0}=30$, the barrier height is decreased from $28 k_{\mathrm{B}} T$ to $14 k_{\mathrm{B}} T$ for $\kappa_{\mathrm{c}}=2$. A change of the stretching modulus parameter $\lambda$ from 5 to 2.5 does not affect the barrier significantly. However, if the bending modulus is decreased by a factor of 2 , the barrier essentially goes away.

\section{Translocation Time}

Using eq 23, the translocation time is calculated for various combinations of values of the parameters. The effect of the external driving potential difference $f_{0}$ on $\tau$ is given in Figure 5 for systems with different elastic moduli and two different vesicle sizes. To calculate the double integral in eq 23 , we sum over numerical values of free energy that we calculated in the previous section for any given value of $a$ and multiply it by the increment value, $\delta$ (i.e., replacing $\int_{0}^{1+\beta} \mathrm{d} \alpha$ with $\delta \sum_{i} F(\alpha=i \delta)$ ). For systems with the same surface characteristics (same elastic moduli), an acceptable range for $f_{0}$ (to get a translocation time between 10 and $10^{5}$ ) is highly dependent on vesicle radius, $r_{0}$. For example, in the case with $\boldsymbol{\kappa}_{\mathrm{c}}=3, \lambda=5$, in order to get a translocation time of 500, we need the driving potential difference, $f_{0}$, to be approximately 15 , if $r_{0}=1.75$. However, only increasing the vesicle radius to $r_{0}=2.5$ will increase the driving potential difference to $f_{0}=70$, in order to get the same translocation time. As is evident from the structure of the equations for the translocation time and the free energy expression, the translocation time should be roughly exponential with the negative of the driving potential difference when the barrier is insignificant. This is exactly what is seen in Figure 5 for the various combinations of $\kappa_{\mathrm{c}}$ and $\lambda$, for each vesicle radius.

The effects of elastic moduli on the translocation time are illustrated in Figure 6. In Figure 6a, the dependence of $\tau$ on the bending modulus $\kappa_{\mathrm{c}}$ is given for two different vesicle radii and two different driving potential differences, by keeping the stretching modulus parameter $\lambda$ as a constant at $\lambda=4$. By keeping $f_{0}$ constant, increasing $\kappa_{\mathrm{c}}$ by a factor of 2 will increase $\tau$ up to 6 orders of magnitude. On the other hand, the effect of $\lambda$ on $\tau$ is weaker, as shown in Figure $6 \mathrm{~b}$. While the translocation time, $\tau$, increases exponentially with $\kappa_{\mathrm{c}}$ and $\lambda$, we see that the effect of $\lambda$ is much weaker in comparison to changes in the bending modulus, $\kappa_{\mathrm{c}}$. Although $\log \tau$ is roughly proportional to both elastic moduli ( $\kappa_{\mathrm{c}}$ and $\lambda$ ), the slope of this law is highly dependent on the vesicle radius, $r_{0}$, and the external potential difference, $f_{0}$.

Finally, we show the effect of the vesicle radius on the translocation time. We find that $\tau$ depends on $r_{0}$ roughly as the exponential of $r_{0}{ }^{2}$, as shown in Figure 7. The linear fit to the numerically calculated results is included in this figure. Here $\kappa_{\mathrm{c}}=1, \lambda=3$, and $f_{0}=43$. The result of $\log \tau$ being proportional to $r_{0}^{2}$ arises from the fact that both the bending and stretching free energies are proportional to the surface area, $r_{0}^{2}$. As has already been pointed 
out through Figure 5, the ratio of the vesicle radius to the pore radius plays a crucial role in controlling the translocation time.

\section{CONCLUSIONS}

We have derived a formula for the translocation time of an incompressible spherical vesicle driven through a narrow pore under an externally applied potential difference in terms of the bending and elastic moduli of the vesicle and its radius, and the radius and length of the pore. In this formulation, we have treated the deformation of the vesicle by the pore within the Helfrich free energy formalism and a constant driving potential difference. The free energy landscape is calculated by considering the three stages of translocation, namely, porefilling, crossing, and pore-depletion stages. Using the thus derived free energy landscape for the translocation process, we have implemented the Fokker-Planck formalism to obtain the average translocation time as the mean first passage time.

In general, there is a free energy barrier for translocation of the vesicle through a narrow pore arising from the obligatory elastic deformation of the vesicle. In the absence of the driving potential difference, the barrier is symmetric in the translocation coordinate and its magnitude depends on the various parameters of the system. For example, the barrier decreases as the spontaneous curvature increases. The free energy barrier is mitigated by the presence of an externally applied potential difference, and the translocation time becomes shorter as the strength of the driving potential difference is increased. The average translocation time depends roughly exponentially on the negative of the driving potential difference. Also, an approximate exponential dependence of the average translocation time on the bending modulus and stretching modulus parameter is observed, although the effect from the bending modulus is very drastic in comparison to that of the stretching modulus. A change in the vesicle size has the biggest effect on the translocation time. The average translocation time depends on the vesicle radius $r_{0}$ as $\exp \left(r_{0}{ }^{2}\right)$.

Indeed, the model presented here is a zeroth-order model in comparison to the biological systems which possess very rich chemical features. In our model, the specific chemical details of the vesicles are parametrized in terms of the material parameters in an effort to extract the universal large scale properties associated with the translocation of a single vesicle through a narrow pore. It is hoped that the above predicted dependencies of translocation kinetics on the elastic properties and size of the vesicle and on the driving potential difference would stimulate experiments on model vesicular systems.

\section{Supplementary Material}

Refer to Web version on PubMed Central for supplementary material.

\section{ACKNOWLEDGMENTS}

This research was supported by NSF Grant No. DMR-1404940 and AFOSR Grant No. FA9550-14-1-0164. 


\section{REFERENCES}

1. Mezei MM, Gulasekharam V. Liposomes-a Selective Drug Delivery System for the Topical Route of Administration I. Lotion Dosage Form. Life Sci. 1980; 26:1473-1477. [PubMed: 6893068]

2. Wachter C, Vierl U, Cevc G. Adaptability and Elasticity of the Mixed Lipid Bilayer Vesicles Containing Non-ionic Surfactant Sesigned for Targeted Drug Delivery across the Skin. J. Drug. Targeting. 2008; 16:611-625.

3. Guo X, Szoka FC. Chemical Approaches to Triggerable Lipid Vesicles for Drug and Gene Delivery. Acc. Chem. Res. 2003; 36:335-341. [PubMed: 12755643]

4. Nelson, D.; Piran, T.; Weinberg, S. Statistical Mechanics of Membranes and Surfaces. 2nd ed.. World Scientific; River Edge, NJ: 2004.

5. Cevc G, Blume G. Lipid Vesicles Penetrate into Intact Skin Owing to the Transdermal Osmotic Gradients and Hydration Force. Biochim. Biophys. Acta, Biomembr. 1992; 1104:226-232.

6. Tordeux C, Fournier J-B. Extravasation of Adhering Vesicles. Europhys. Lett. 2002; 60:875-881.

7. Dietrich C, Angelova M, Pouligny B. Adhesion of Latex Spheres to Giant Phospholipid Vesicles: Statics and Dynamics. J. Phys. II. 1997; 7:1651-1682.

8. Gompper G, Kroll DM. Driven Transport of Fluid Vesicles through Narrow Pores. Phys. Rev. E: Stat. Phys., Plasmas, Fluids, Relat. Interdiscip. Top. 1995; 52:4198-4208.

9. Linke GT, Lipowsky R, Gruhn T. Osmotically Induced Passage of Vesicles through Narrow Pores. Europhys. Lett. 2006; 74:916-922.

10. Deserno M, Gelbart WM. Adhesion and Wrapping in Colloid-vesicle Complexes. J. Phys. Chem. B. 2002; 106:5543-5552.

11. Deserno M. Elastic Deformation of a Fluid Membrane upon Colloid Binding. Phys. Rev. E. 2004; 69:031903.

12. Tu ZC, Ou-Yang ZC. A Geometric Theory on the Elasticity of Bio-membranes. J. Phys. A: Math. Gen. 2004; 37:11407-11429.

13. Seifert U. Configurations of Fluid Membranes and Vesicles. Adv. Phys. 1997; 46:13-137.

14. Fleck CC, Netz RR. Electrostatic Colloid-membrane Binding. Europhys. Lett. 2004; 67:314-320.

15. Seifert U. Vesicles of Toroidal Topology. Phys. Rev. Lett. 1991; 66:2404-2407. [PubMed: 10043476]

16. Jie Y, Quanhui L, Zhong-Can O. Numerical Observation of Nonaxisymmetric Vesicles in Fluid Membranes. Phys. Rev. E: Stat. Phys., Plasmas, Fluids, Relat. Interdiscip. Top. 1998; 58:4730_ 4736.

17. Olbrich K, Rawicz W, Needham D, Evans E. Water Permeability and Mechanical Strength of Polyunsaturated Lipid Bilayers. Biophys. J. 2000; 79:321-327. [PubMed: 10866958]

18. Deuling HJ, Helfrich W. The Curvature Elasticity of Fluid Membranes: a Catalogue of Vesicle Shapes. J. Phys. (Paris). 1976; 37:1335-1345.

19. Lipowsky R. The Conformation of Membranes. Nature. 1991; 349:475-481. [PubMed: 1992351]

20. Mirigian S, Muthukumar M. Kinetics of Particle Wrapping by a Vesicle. J. Chem. Phys. 2013; 139:044908. [PubMed: 23902020]

21. Fournier J-B, Galatola P. Corrections to the Laplace Law for Vesicle Aspiration in Micropipettes and other Confined Geometries. Soft Matter. 2008; 4:2463-2470.

22. Helfrich W. Elastic Properties of Lipid Bilayers: Theory and Possible Experiments. Z. Naturforsch., C. 1973; 28:693-703. [PubMed: 4273690]

23. Israelachvili, JN. Intermolecular and Surface Forces. 3rd ed.. Academic Press; Waltham, MA: 2011.

24. Szleifer I, Kramer D, Ben-Shaul A, Gelbart WM, Safran SA. Molecular Theory of Curvature Elasticity in Surfactant Films. J. Chem. Phys. 1990; 92:6800-6817.

25. Evans E. Bending Resistance and Chemically Induced Moments in Membrane Bilayers. Biophys. J. 1974; 14:923-931. [PubMed: 4429770]

26. Rand RP, Fuller NL, Gruner SM, Parsegian VA. Membrane Curvature, Lipid Segregation, and Structural Transitions for Phospholipids under Dual-solvent Stress. Biochemistry. 1990; 29:76-87. [PubMed: 2322550] 
27. Muthukumar, M. Polymer Translocation. CRC Press; Boca Raton, FL: 2011.

28. Risken, H. Fokker-Planck Equation. Springer; New York: 1984.

29. Kummrow M, Helfrich W. Deformation of Giant Lipid Vesicles by Electric Fields. Phys. Rev. A: At., Mol., Opt. Phys. 1991; 44:8356-8360.

30. Evans E, Heinrich V, Ludvig F, Rawicz W. Dynamic Tension Spectroscopy and Strength of Biomembranes. Biophys. J. 2003; 85:2342-2350. [PubMed: 14507698]

31. Loftus AF, Noreng S, Hsieh VL, Parthasarathy R. Robust Measurement of Membrane Bending Moduli Using Light Sheet Fluorescence Imaging of Vesicle Fluctuations. Langmuir. 2013;

29:14588-14594. [PubMed: 24180269] 

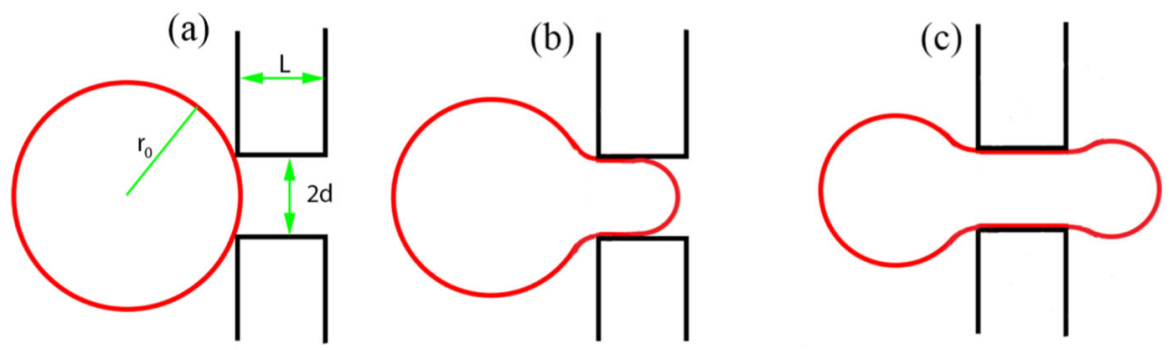

(d)
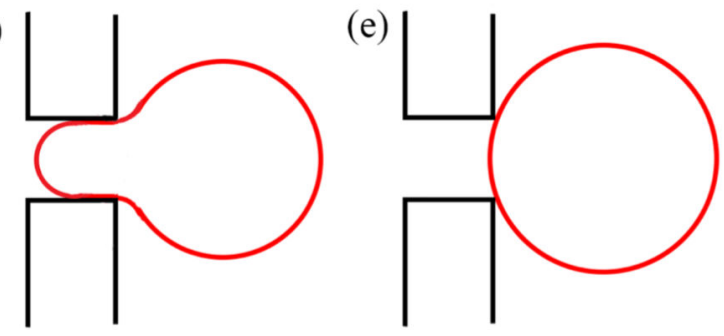

Figure 1.

Schematics of the translocation of a spherical vesicle of initial radius $r_{0}$ through a narrow pore of radius $d$ and length $L$. (a) Initial state of the vesicle in the donor compartment, (b) partial penetration of the vesicle into the pore, (c) filling of the pore with the remainder of the vesicle partitioned into both the donor and receiver compartments, (d) partial filling of the pore in the exit stage, and (e) final state of the vesicle in the receiver compartment. 

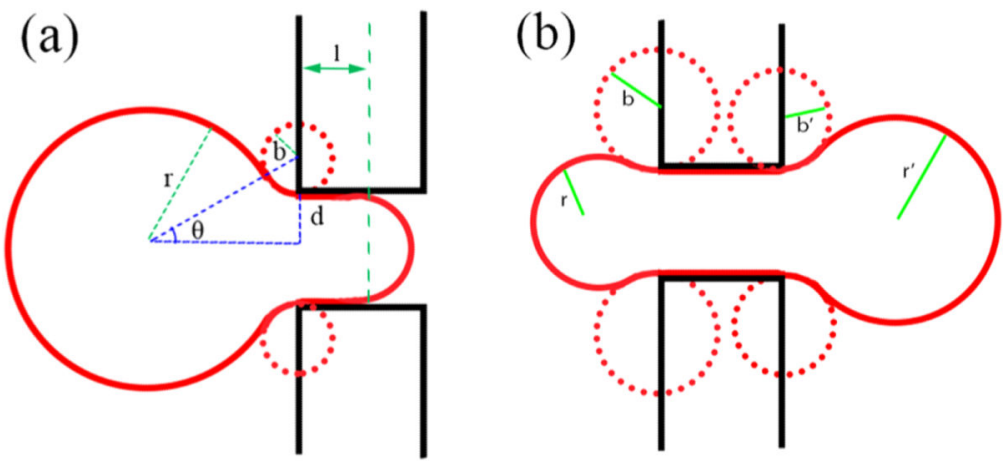

Figure 2.

Various parameters used to define the free energy of the deformed vesicle. (a) Filling stage: $r$ is the radius of the partial sphere, $b$ is the small radius of the toroidal vesicle. Dotted lines show the cross section of a hypothetical torus that keeps the curvature of the vesicle smooth. $\theta$ is the angle between the center of the spherical part and the center of the small toroidal ring. (b) Crossing stage: Two toroids are needed. $b^{\prime}, r^{\prime}$, and $\theta^{\prime}$ correspond to the toroid on the receiver side, and $b, r$, and $\theta$ correspond to the donor side as in part a. 


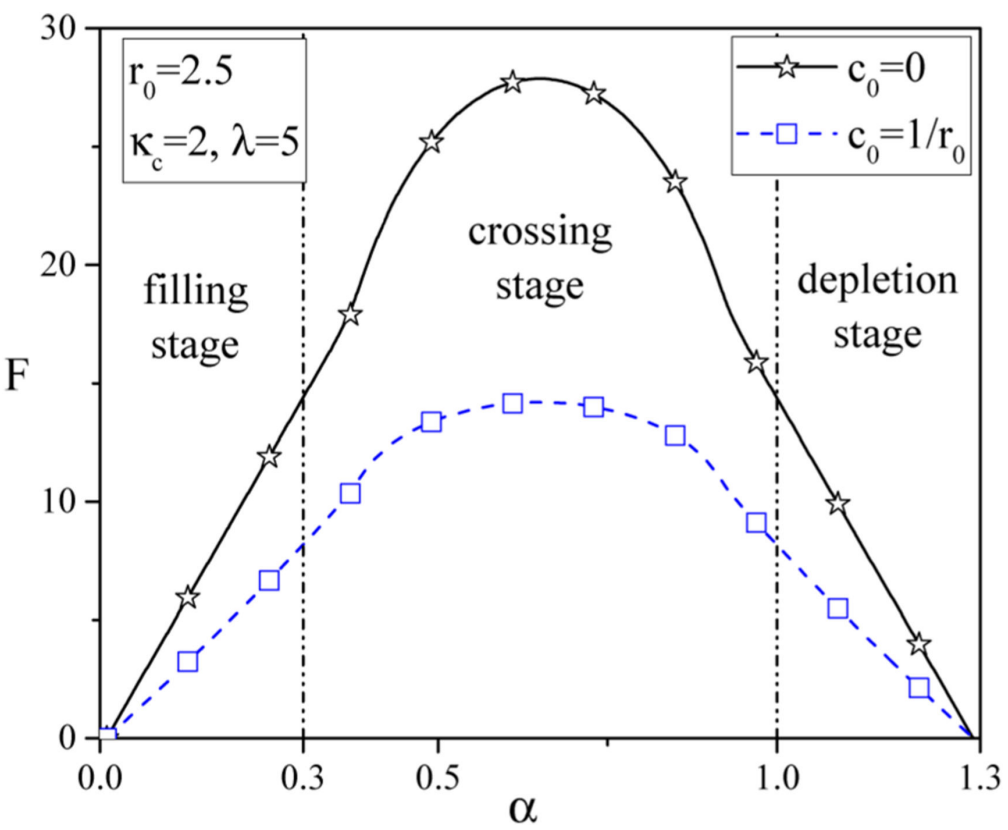

Figure 3.

Helfrich free energy for $r_{0}=2.5 d, \beta=0.3 . \kappa_{\mathrm{c}}=2$ and $\lambda=5$. The solid line (black) is without spontaneous curvature. The dashed line (blue) is with existence of $c_{0}$. The three stages of translocation are also marked. 


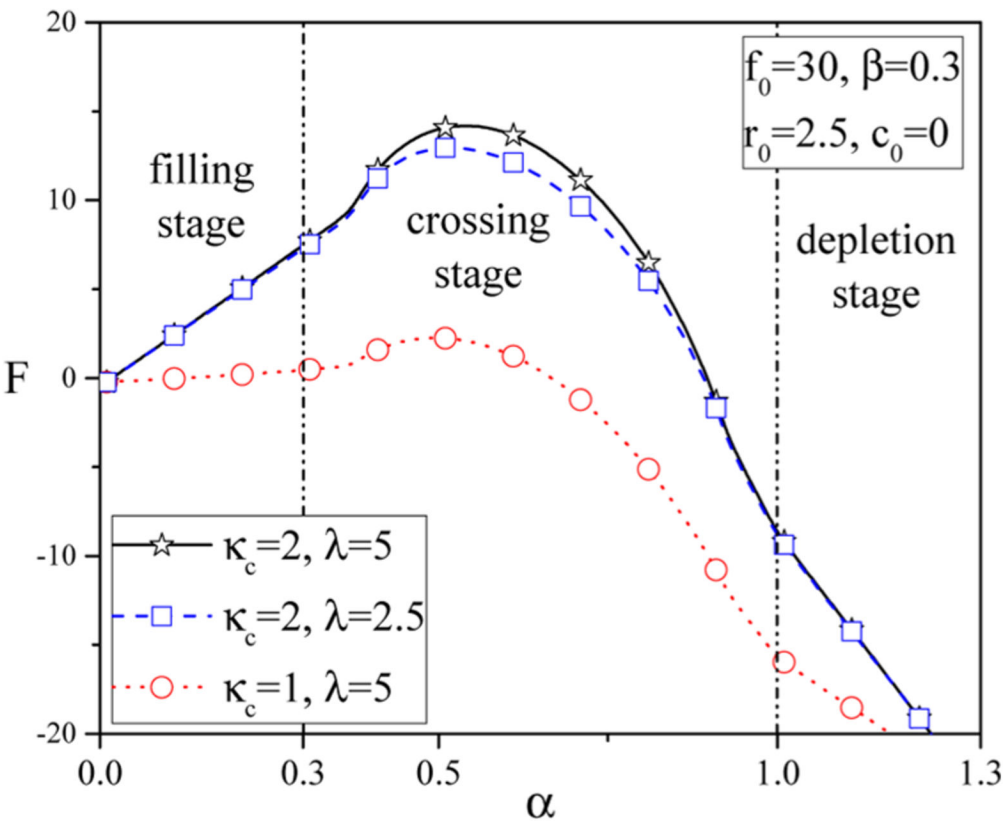

Figure 4.

Free energy in the presence of external field for $r_{0}=2.5 d, \beta=0.3$. Red (dotted) is for $\kappa_{\mathrm{c}}=1$ and $\lambda=5$. Blue (dashed) is for $\kappa_{\mathrm{c}}=2$ and $\lambda=2.5$. Black (solid) is for $\kappa_{\mathrm{c}}=2$ and $\lambda=5 . c_{0}=$ 0 and $f_{0}=30$. 

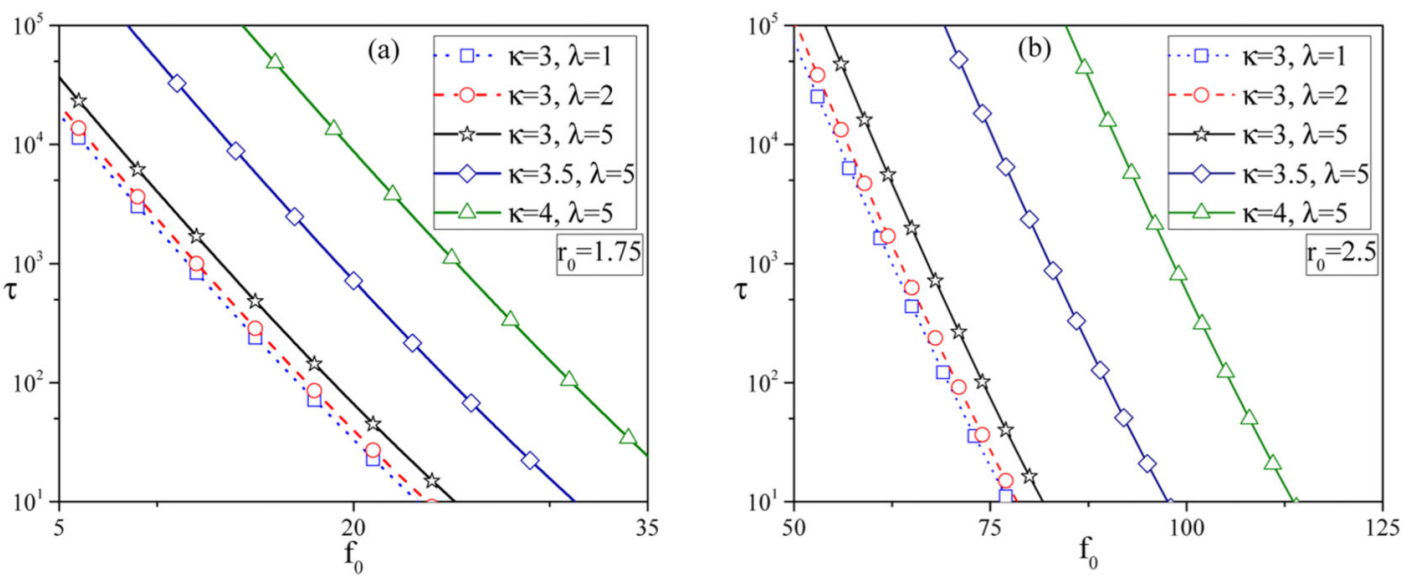

Figure 5.

Effect of driving force for different values of $\kappa_{\mathrm{c}}$ and $\lambda$ : (a) $r_{0}=1.75$; (b) $r_{0}=2.5$. 

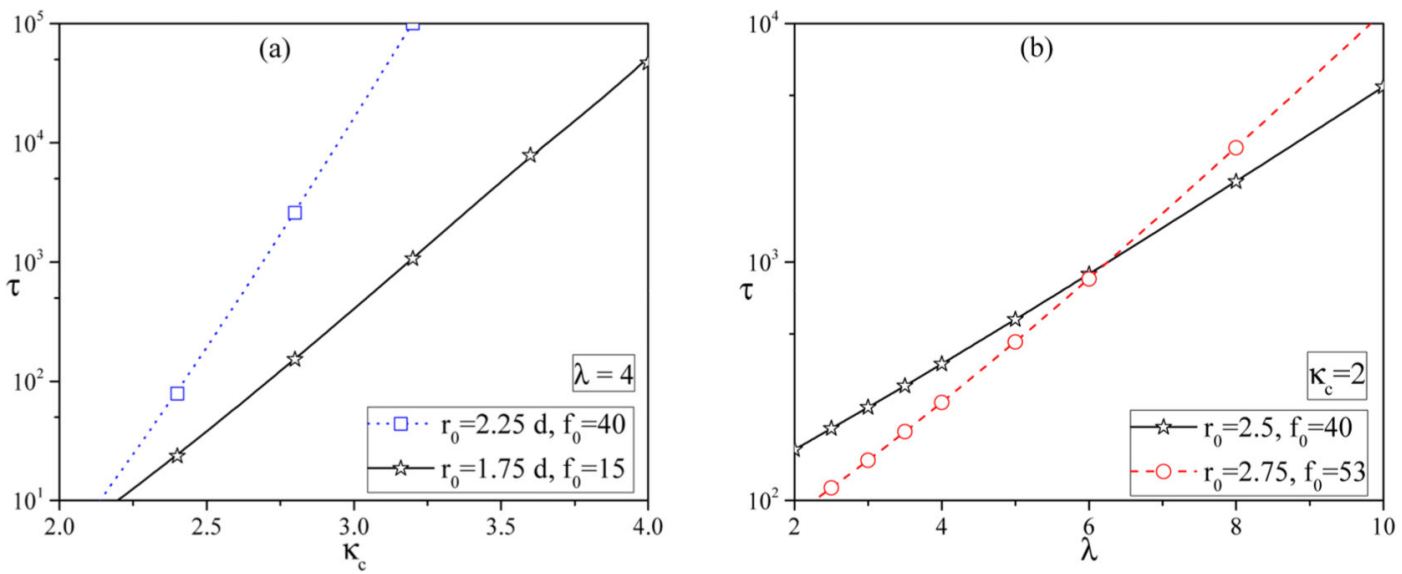

Figure 6.

Effect of elastic moduli for different vesicle sizes with appropriate external forces: (a)

Translocation time as a function of $\kappa_{\mathrm{c}}$; by keeping $f_{0}$ constant, increasing $\kappa_{\mathrm{c}}$ by a factor of 2 will increase $\tau$ up to 6 orders of magnitude. (b) Translocation time as a function of $\lambda$; changing $\lambda$ from 2 to 10 will change $\tau$ almost 2 orders of magnitude. 


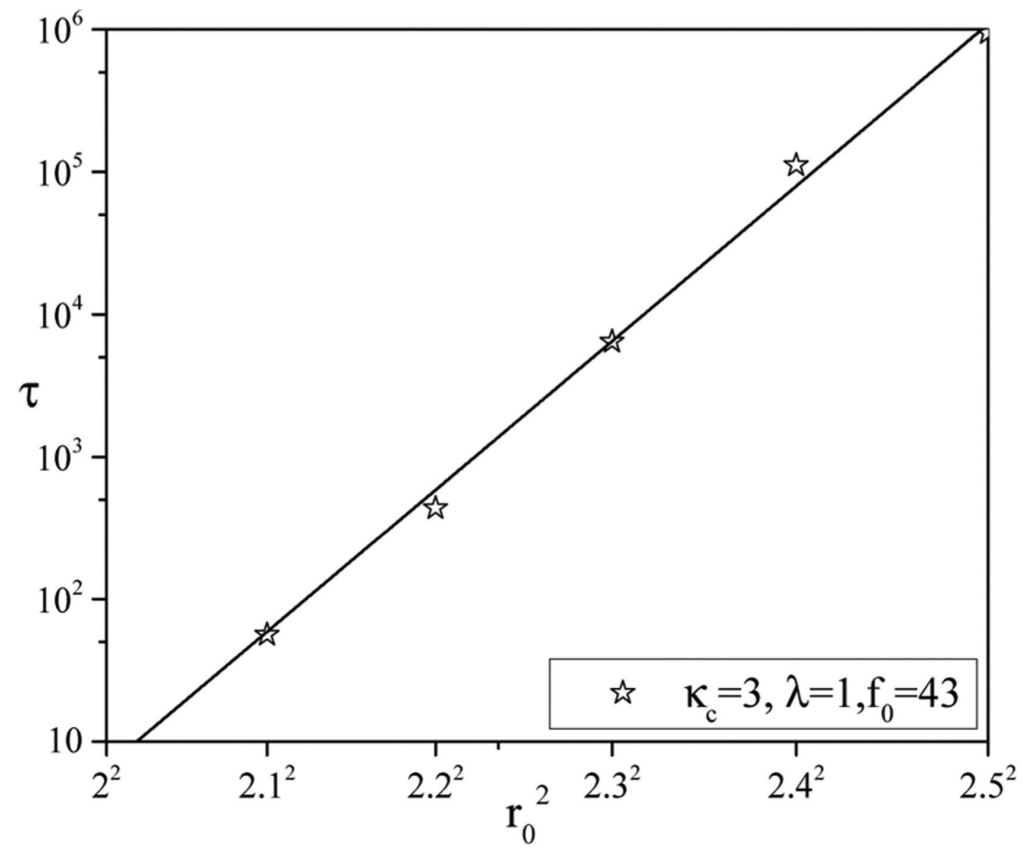

Figure 7.

Effect of vesicle radius compared to pore entrance as a function of $r_{0}^{2} ; \kappa_{\mathrm{c}}=1, \lambda=3$, and $f_{0}$ $=43$. The straight line is the linear fit to the data. 